\title{
Stability analysis of dynamic thin shells
}

\author{
Francisco S. N. Lobo $\nmid$ and Paulo Crawford $\ddagger$ \\ Centro de Astronomia e Astrofísica da Universidade de Lisboa, \\ Campo Grande, Ed. C8 1749-016 Lisboa, Portugal
}

\begin{abstract}
.
We analyze the stability of generic spherically symmetric thin shells to linearized perturbations around static solutions. We include the momentum flux term in the conservation identity, deduced from the "ADM" constraint and the Lanczos equations. Following the Ishak-Lake analysis, we deduce a master equation which dictates the stable equilibrium configurations. Considering the transparency condition, we study the stability of thin shells around black holes, showing that our analysis is in agreement with previous results. Applying the analysis to traversable wormhole geometries, by considering specific choices for the form function, we deduce stability regions, and find that the latter may be significantly increased by considering appropriate choices for the redshift function.

PACS numbers: 04.20.Cv, 04.20.Gz, 04.70.Bw
\end{abstract}

$\dagger$ flobo@cosmo.fis.fc.ul.pt

$\ddagger$ crawford@cosmo.fis.fc.ul.pt 


\section{Introduction}

The study of hypersurfaces of discontinuity plays a fundamental role in general relativity. Pioneering work can be traced back to Sen [1], Lanczos [2], Darmois [3], and later by O'Brien and Synge [4, Lichnerowicz [5], and Israel [6], amongst others. Several approaches to deduce the geometric conditions of the surface layer were carried out. For instance, Sen in a relatively unknown paper [1, used continuous four-dimensional coordinates across the layer, an approach later applied by Lichnerowicz, and O'Brien and Synge. Darmois expressed the surface properties of the discontinuity of the extrinsic curvature across the layer as a function of the surfaces intrinsic coordinates, which was later generalized by Israel [6], using independently defined four-dimensional coordinate systems $x_{ \pm}^{\mu}$ of the two different manifolds glued at the junction surface. Since then, the hypersurfaces of discontinuity have had an extensive range of applicability. It is perhaps important to emphasize that the matching of an interior dust solution to an exterior Schwarzschild spacetime, by Oppenheimer and Snyder, provided the first insights of the gravitational collapse into a black hole [7]. One can mention more recent applications of the thin shell formalism, namely, gravitational collapse of radiating shells, the evolution of bubbles and domain walls in cosmological settings and in inflationary models, the structure and dynamics of voids in the large scale structure of the universe, shells around black holes and their respective stability, signature changes, matchings of cosmological solutions, and applications to the Randall-Sundrum brane world scenario to wormhole physics. An analysis in thick gravitating walls expanded in powers of the thickness of the wall has also been carried out [8, 9]. The lightlike limit was analyzed in Ref. [10, and an equivalence between the Darmois-Israel formalism and the distributional method for thin shells was established in Ref. [11. Due to the extensive applications of the DarmoisIsrael formalism, a computer algebra system was implemented to aid relativists in the evaluation of junction conditions and the parameters associated with thin shells [12].

The application of the Darmois-Israel formalism to black holes and wormhole physics is of a particular interest. Relatively to black holes, in an interesting paper [13, the Schwarzschild metric inside the event horizon was matched to the de Sitter solution at a spacelike junction surface. The respective properties of the layer were explored, and it was found that instead of a singularity, a closed world can be formed inside the black hole. In Ref. [14], a thin shell was constructed around a black hole and its characteristics were explored by imposing the energy conditions, and in Ref. 15. the respective stability of the thin shell was analyzed against spherically symmetric perturbations about a static solution. Relatively to wormhole physics, using the cutand-paste technique, Visser constructed thin-shell wormholes, and considered a partial stability analysis by imposing specific equations of state [16, 17, 18, 19]. In Ref. [20] thinshell Schwarzschild wormholes were considered and the respective stability to spherically symmetric perturbations around static solutions was analyzed, in the spirit of [15]. It was later found that the inclusion of a charge [21] and of a positive cosmological constant 22] significantly increases the stability regions. Specific wormhole solutions were also 
constructed by matching an interior traversable wormhole solution to exterior vacuum spacetimes at a junction interface [23, 24, 25, 26]. Thin shells with a zero surface energy density were analyzed in Ref. [23], dust shells in Ref. [24], generic surface stresses in Ref. [25, and a similar analysis for plane symmetric traversable wormholes in an anti-de Sitter background was extensively studied in Ref. [26].

Ishak and Lake developed a formalism to analyze the stability of spherically symmetric thin shells by imposing spacetimes that satisfy the transparency condition [27, which amounts to considering solutions that do not contribute with the discontinuity flux term in the conservation identity. The dynamics of timelike spherical thin shells, satisfying the transparency condition were also analyzed in Ref. 28. In this work, we include this momentum flux term, which severely complicates the analysis, and following the Ishak-Lake approach, we analyze the stability of generic spherically symmetric thin shells to linearized perturbations about static equilibrium solutions. We then deduce a master equation dictating the stability regions. Considering the transparency condition, we study the stability of thin shells around black holes, showing that our analysis is in agreement with previous results. We also apply the general formalism to specific wormhole solutions, and by considering particular form functions, we verify that the stability regions can be significantly increased by taking into account appropriate choices of the redshift function. In this context, a specific application of the general formalism developed in this paper has been applied to traversable wormhole solutions [29] supported by phantom energy [30, 31].

The plan of this paper is as follows: In Section II, we outline a brief summary of the Darmois-Israel formalism, which shall be used throughout the work. In Section III, we present two generic spherically symmetric spacetimes matched together at a junction interface and deduce the surface stresses of the thin shell. Based on the inclusion of the momentum flux term in the conservation identity, fundamental relationships for analyzing the stability regions are also deduced. From the equation of motion, a master equation, dictating the stable equilibrium configurations, is deduced. In Section IV, we shall apply the formalism developed to thin shells around black holes. In Section V, the formalism is applied to traversable wormholes, imposing several choices for the form and redshift functions. Finally, in Section VI, we conclude.

\section{The Darmois-Israel formalism}

Consider two distinct spacetime manifolds, $\mathcal{M}_{+}$and $\mathcal{M}_{-}$, with metrics given by $g_{\mu \nu}^{+}\left(x_{+}^{\mu}\right)$ and $g_{\mu \nu}^{-}\left(x_{-}^{\mu}\right)$, in terms of independently defined coordinate systems $x_{+}^{\mu}$ and $x_{-}^{\mu}$. The manifolds are bounded by hypersurfaces $\Sigma_{+}$and $\Sigma_{-}$, respectively, with induced metrics

$g_{i j}^{+}$and $g_{i j}^{-}$. The hypersurfaces are isometric, i.e., $g_{i j}^{+}(\xi)=g_{i j}^{-}(\xi)=g_{i j}(\xi)$, in terms of the intrinsic coordinates, invariant under the isometry. A single manifold $\mathcal{M}$ is obtained by gluing together $\mathcal{M}_{+}$and $\mathcal{M}_{-}$at their boundaries, i.e., $\mathcal{M}=\mathcal{M}_{+} \cup \mathcal{M}_{-}$, with the natural identification of the boundaries $\Sigma=\Sigma_{+}=\Sigma_{-}$. In particular, assuming the continuity of the four-dimensional coordinates $x_{ \pm}^{\mu}$ across $\Sigma$, then $g_{\mu \nu}^{-}=g_{\mu \nu}^{+}$is required, which together 
with the continuous derivatives of the metric components $\partial g_{\mu \nu} /\left.\partial x^{\alpha}\right|_{-}=\partial g_{\mu \nu} /\left.\partial x^{\alpha}\right|_{+}$, provide the Lichnerowicz conditions [5].

The three holonomic basis vectors $\mathbf{e}_{(i)}=\partial / \partial \xi^{i}$ tangent to $\Sigma$ have the following components $\left.e_{(i)}^{\mu}\right|_{ \pm}=\partial x_{ \pm}^{\mu} / \partial \xi^{i}$, which provide the induced metric on the junction surface by the following scalar product

$$
g_{i j}=\mathbf{e}_{(i)} \cdot \mathbf{e}_{(j)}=\left.g_{\mu \nu} e_{(i)}^{\mu} e_{(j)}^{\nu}\right|_{ \pm} .
$$

We shall consider a timelike junction surface $\Sigma$, defined by the parametric equation of the form $f\left(x^{\mu}\left(\xi^{i}\right)\right)=0$. The unit normal 4 -vector, $n^{\mu}$, to $\Sigma$ is defined as

$$
n_{\mu}= \pm\left|g^{\alpha \beta} \frac{\partial f}{\partial x^{\alpha}} \frac{\partial f}{\partial x^{\beta}}\right|^{-1 / 2} \frac{\partial f}{\partial x^{\mu}},
$$

with $n_{\mu} n^{\mu}=+1$ and $n_{\mu} e_{(i)}^{\mu}=0$. The Israel formalism requires that the normals point from $\mathcal{M}_{-}$to $\mathcal{M}_{+}[6]$.

The extrinsic curvature, or the second fundamental form, is defined as $K_{i j}=$ $n_{\mu ; \nu} e_{(i)}^{\mu} e_{(j)}^{\nu}$, or

$$
K_{i j}^{ \pm}=-n_{\mu}\left(\frac{\partial^{2} x^{\mu}}{\partial \xi^{i} \partial \xi^{j}}+\Gamma_{\alpha \beta}^{\mu \pm} \frac{\partial x^{\alpha}}{\partial \xi^{i}} \frac{\partial x^{\beta}}{\partial \xi^{j}}\right) .
$$

Note that for the case of a thin shell $K_{i j}$ is not continuous across $\Sigma$, so that for notational convenience, the discontinuity in the second fundamental form is defined as $\kappa_{i j}=K_{i j}^{+}-K_{i j}^{-}$. In particular, the condition that $g_{i j}^{-}=g_{i j}^{+}$, together with the continuity of the extrinsic curvatures across $\Sigma, K_{i j}^{-}=K_{i j}^{+}$, provide the Darmois conditions [3].

Now, the Lanczos equations follow from the Einstein equations for the hypersurface, and are given by

$$
S_{j}^{i}=-\frac{1}{8 \pi}\left(\kappa_{j}^{i}-\delta_{j}^{i} \kappa_{k}^{k}\right),
$$

where $S_{j}^{i}$ is the surface stress-energy tensor on $\Sigma$.

The first contracted Gauss-Kodazzi equation or the "Hamiltonian" constraint

$$
G_{\mu \nu} n^{\mu} n^{\nu}=\frac{1}{2}\left(K^{2}-K_{i j} K^{i j}-{ }^{3} R\right),
$$

with the Einstein equations provide the evolution identity

$$
S^{i j} \bar{K}_{i j}=-\left[T_{\mu \nu} n^{\mu} n^{\nu}\right]_{-}^{+} .
$$

The convention $\left.[X]_{-}^{+} \equiv X^{+}\right|_{\Sigma}-\left.X^{-}\right|_{\Sigma}$ and $\bar{X} \equiv\left(\left.X^{+}\right|_{\Sigma}+\left.X^{-}\right|_{\Sigma}\right) / 2$ is used.

The second contracted Gauss-Kodazzi equation or the "ADM" constraint

$$
G_{\mu \nu} e_{(i)}^{\mu} n^{\nu}=K_{i \mid j}^{j}-K_{, i}
$$

with the Lanczos equations gives the conservation identity

$$
S_{j \mid i}^{i}=\left[T_{\mu \nu} e_{(j)}^{\mu} n^{\nu}\right]_{-}^{+} .
$$

In particular, considering spherical symmetry considerable simplifications occur, namely $\kappa_{j}^{i}=\operatorname{diag}\left(\kappa_{\tau}^{\tau}, \kappa_{\theta}^{\theta}, \kappa_{\theta}^{\theta}\right)$. The surface stress-energy tensor may be written 
in terms of the surface energy density, $\sigma$, and the surface pressure, $\mathcal{P}$, as $S_{j}^{i}=$ $\operatorname{diag}(-\sigma, \mathcal{P}, \mathcal{P})$. The Lanczos equations then reduce to

$$
\begin{aligned}
\sigma & =-\frac{1}{4 \pi} \kappa_{\theta}^{\theta}, \\
\mathcal{P} & =\frac{1}{8 \pi}\left(\kappa_{\tau}^{\tau}+\kappa_{\theta}^{\theta}\right) .
\end{aligned}
$$

\section{Generic dynamic spherically symmetric thin shells}

\subsection{Junction conditions}

We shall consider, in particular, the matching of two static and spherically symmetric spacetimes given by the following line elements

$$
d s_{ \pm}^{2}=-e^{2 \alpha_{ \pm}\left(r_{ \pm}\right)} d t_{ \pm}^{2}+e^{2 \beta_{ \pm}\left(r_{ \pm}\right)} d r_{ \pm}^{2}+r_{ \pm}^{2}\left(d \theta_{ \pm}^{2}+\sin ^{2} \theta_{ \pm} d \phi_{ \pm}^{2}\right)
$$

of $\mathcal{M}_{ \pm}$, respectively. Using the Einstein field equation, $G_{\hat{\mu} \hat{\nu}}=8 \pi T_{\hat{\mu} \hat{\nu}}$, in an orthonormal reference frame, (with $c=G=1$ ) the stress-energy tensor components are given by

$$
\begin{aligned}
& \rho(r)=\frac{1}{8 \pi} \frac{e^{-2 \beta}}{r^{2}}\left(2 \beta^{\prime} r+e^{2 \beta}-1\right), \\
& p_{r}(r)=\frac{1}{8 \pi} \frac{e^{-2 \beta}}{r^{2}}\left(2 \alpha^{\prime} r-e^{2 \beta}+1\right), \\
& p_{t}(r)=\frac{1}{8 \pi} \frac{e^{-2 \beta}}{r}\left[-\beta^{\prime}+\alpha^{\prime}+r \alpha^{\prime \prime}+r\left(\alpha^{\prime}\right)^{2}-r \alpha^{\prime} \beta^{\prime}\right],
\end{aligned}
$$

where we have dropped the \pm subscripts as not to overload the notation, and in which $\rho(r)$ is the energy density, $p_{r}(r)$ is the radial pressure, and $p_{t}(r)$ is the lateral pressure measured in the orthogonal direction to the radial direction.

The energy conditions will play an important role in the analysis that follows, so we will at this stage define the null energy condition (NEC). The latter is satisfied if $T_{\mu \nu} k^{\mu} k^{\nu} \geq 0$, where $T_{\mu \nu}$ is the stress-energy tensor and $k^{\mu}$ any null vector. Along the radial direction, with $k^{\hat{\mu}}=(1, \pm 1,0,0)$ in the orthonormal frame, we then have the following condition

$$
T_{\hat{\mu} \hat{\nu}} k^{\hat{\mu}} k^{\hat{\nu}}=\rho(r)+p_{r}(r)=\frac{1}{4 \pi r} e^{-2 \beta}\left(\alpha^{\prime}+\beta^{\prime}\right) \geq 0 .
$$

The single manifold, $\mathcal{M}$, is obtained by gluing $\mathcal{M}_{+}$and $\mathcal{M}_{-}$at $\Sigma$, i.e., at $f(r, \tau)=r-a(\tau)=0$. In order for these line elements to be continuous across the junction, we impose the following coordinate transformations

$t_{+}=\frac{e^{\alpha_{-}(a)}}{e^{\alpha_{+}(a)}} t_{-},\left.\quad \frac{d r_{+}}{d r_{-}}\right|_{r=a}=\frac{e^{\beta_{-}(a)}}{e^{\beta_{+}(a)}}, \quad \theta_{+}=\theta_{-} \quad$ and $\quad \phi_{+}=\phi_{-}$.

The intrinsic metric to $\Sigma$ is thus provided by

$$
d s_{\Sigma}^{2}=-d \tau^{2}+a(\tau)^{2}\left(d \theta^{2}+\sin ^{2} \theta d \phi^{2}\right) .
$$

The imposition of spherical symmetry is sufficient to conclude that there is no

gravitational radiation, independently of the behavior of the junction surface. The 
position of the junction surface is given by $x^{\mu}(\tau, \theta, \phi)=(t(\tau), a(\tau), \theta, \phi)$, and the respective 4 -velocity is

$$
U_{ \pm}^{\mu}=\left(e^{\beta_{ \pm}(a)-\alpha_{ \pm}(a)} \sqrt{e^{-2 \beta_{ \pm}(a)}+\dot{a}^{2}}, \dot{a}, 0,0\right),
$$

where the overdot denotes a derivative with respect to $\tau$.

The unit normal to the junction surface may be determined by equation (2) or by the contractions, $U^{\mu} n_{\mu}=0$ and $n^{\mu} n_{\mu}=+1$, and is given by

$$
n_{ \pm}^{\mu}=\left(e^{\beta_{ \pm}(a)-\alpha_{ \pm}(a)} \dot{a}, \sqrt{e^{-2 \beta_{ \pm}(a)}+\dot{a}^{2}}, 0,0\right)
$$

Using equation (3), the non-trivial components of the extrinsic curvature are given by

$$
\begin{aligned}
& K_{\theta}^{\theta \pm}=\frac{1}{a} \sqrt{e^{-2 \beta_{ \pm}}+\dot{a}^{2}}, \\
& K_{\tau}^{\tau \pm}=\frac{\alpha_{ \pm}^{\prime}\left(e^{-2 \beta_{ \pm}}+\dot{a}^{2}\right)+\ddot{a}+\beta_{ \pm}^{\prime} \dot{a}^{2}}{\sqrt{e^{-2 \beta_{ \pm}}+\dot{a}^{2}}} .
\end{aligned}
$$

The Einstein equations, eqs. (91)-(10), with the extrinsic curvatures, eqs. (20)-(21), then provide us with the following expressions for the surface stresses

$$
\begin{aligned}
& \sigma=-\frac{1}{4 \pi a}\left[\sqrt{e^{-2 \beta}+\dot{a}^{2}}\right]_{-}^{+}, \\
& \mathcal{P}=\frac{1}{8 \pi a}\left[\frac{\left(1+a \alpha^{\prime}\right)\left(e^{-2 \beta}+\dot{a}^{2}\right)+a \ddot{a}+\beta^{\prime} a \dot{a}^{2}}{\sqrt{e^{-2 \beta}+\dot{a}^{2}}}\right]_{-}^{+} .
\end{aligned}
$$

If the surface stress-energy terms are zero, the junction is denoted as a boundary surface. If surface stress terms are present, the junction is called a thin shell. Note that the surface mass of the thin shell is given by $m_{s}=4 \pi a^{2} \sigma$

\subsection{Conservation identity}

Taking into account the transparency condition, $\left[G_{\mu \nu} U^{\mu} n^{\nu}\right]_{-}^{+}=0$, the conservation identity, equation (8), provides the simple relationship

$$
\dot{\sigma}=-2 \frac{\dot{a}}{a}(\sigma+\mathcal{P})
$$

or

$$
\frac{d(\sigma A)}{d \tau}+\mathcal{P} \frac{d A}{d \tau}=0
$$

where $A=4 \pi a^{2}$ is the area of the thin shell. The first term represents the variation of the internal energy of the shell, and the second term is the work done by the shell's internal force.

Now, taking into account the momentum flux term in equation (8),$\left[T_{\mu \nu} e_{(\tau)}^{\mu} n^{\nu}\right]_{-}^{+}=$ $\left[T_{\mu \nu} U^{\mu} n^{\nu}\right]_{-}^{+}$, we have

$$
\left[T_{\mu \nu} U^{\mu} n^{\nu}\right]_{-}^{+}=\left[\left(T_{\hat{t} \hat{t}}+T_{\hat{r} \hat{r}}\right) e^{2 \beta} \dot{a} \sqrt{e^{-2 \beta}+\dot{a}^{2}}\right]_{-}^{+},
$$


where $T_{\hat{t} \hat{t}}$ and $T_{\hat{r} \hat{r}}$ are the stress-energy tensor components given in an orthonormal basis. The flux term, equation (26), corresponds to the net discontinuity in the momentum flux $F_{\mu}=T_{\mu \nu} U^{\nu}$ which impinges on the shell. Thus in the general case, the conservation identity provides the following relationship

$$
\sigma^{\prime}=-\frac{2}{a}(\sigma+\mathcal{P})+\Xi
$$

where for notational convenience, we have defined $\Xi$ as

$$
\Xi=\left[\left(T_{\hat{t} \hat{t}}+T_{\hat{r} \hat{r}}\right) e^{2 \beta} \sqrt{e^{-2 \beta}+\dot{a}^{2}}\right]_{-}^{+}=\frac{1}{4 \pi a}\left[\left(\alpha^{\prime}+\beta^{\prime}\right) \sqrt{e^{-2 \beta}+\dot{a}^{2}}\right]_{-}^{+} .
$$

Note that this flux term vanishes in the particular case of $p=-\rho$.

Taking into account the following relationship

$$
\sigma+\mathcal{P}=\frac{1}{8 \pi a}\left[\frac{\left(a \alpha^{\prime}-1\right) e^{-2 \beta}+a\left(\alpha^{\prime}+\beta^{\prime}\right) \dot{a}^{2}-\dot{a}^{2}+a \ddot{a}}{\sqrt{e^{-2 \beta}+\dot{a}^{2}}}\right]_{-}^{+},
$$

and the definition of $\Xi$, equation (27) takes the form

$$
\sigma^{\prime}=\frac{1}{4 \pi a^{2}}\left[\frac{\left(1+a \beta^{\prime}\right) e^{-2 \beta}+\dot{a}^{2}-a \ddot{a}}{\sqrt{e^{-2 \beta}+\dot{a}^{2}}}\right]_{-}^{+},
$$

which can also be obtained by taking the radial derivative of equation (22). Evaluated at the static solution $a_{0}$, with $\dot{a}=\ddot{a}=0$, this reduces to

$$
\sigma^{\prime}\left(a_{0}\right)=\frac{1}{4 \pi a_{0}^{2}}\left[e^{-\beta}\left(1+a_{0} \beta^{\prime}\right)\right]_{-}^{+} .
$$

Now, using $m_{s}=4 \pi a^{2} \sigma$, and taking into account the radial derivative of $\sigma^{\prime}$, equation (27) can be rearranged to provide the following relationship

$$
\left(\frac{m_{s}}{2 a}\right)^{\prime \prime}=\bar{\Upsilon}-4 \pi \sigma^{\prime} \eta
$$

with the parameter $\eta$ defined as $\eta=\mathcal{P}^{\prime} / \sigma^{\prime}$, and $\bar{\Upsilon}$ given by

$$
\bar{\Upsilon} \equiv \frac{4 \pi}{a}(\sigma+\mathcal{P})+2 \pi a \Xi^{\prime}
$$

The physical interpretation of $\eta$ is extensively discussed in [20, 27], and $\sqrt{\eta}$ is normally interpreted as the speed of sound. Equation (32) will play a fundamental role in determining the stability regions of the respective solutions which will be analyzed further ahead.

For self-completeness, we shall also add the expression of $\Xi^{\prime}$, given by

$\Xi^{\prime}=\frac{1}{4 \pi a^{2}}\left[\left[a\left(\alpha^{\prime \prime}+\beta^{\prime \prime}\right)-\left(\alpha^{\prime}+\beta^{\prime}\right)\right] \sqrt{e^{-2 \beta}+\dot{a}^{2}}+\frac{a\left(\alpha^{\prime}+\beta^{\prime}\right)\left(-\beta^{\prime} e^{-2 \beta}+\ddot{a}\right)}{\sqrt{e^{-2 \beta}+\dot{a}^{2}}}\right]_{-}^{+}$,

which evaluated at the static solution, $a_{0}$, reduces to

$$
\Xi_{0}^{\prime}=\frac{1}{4 \pi a_{0}^{2}}\left[\left[a_{0}\left(\alpha^{\prime \prime}+\beta^{\prime \prime}\right)-\left(\alpha^{\prime}+\beta^{\prime}\right)\left(1+a_{0} \beta^{\prime}\right)\right] e^{-\beta}\right]_{-}^{+},
$$




\subsection{Equation of motion}

Rearranging equation (22) into the form

$$
\sqrt{e^{-2 \beta_{+}}+\dot{a}^{2}}=\sqrt{e^{-2 \beta_{-}}+\dot{a}^{2}}-4 \pi a \sigma,
$$

we deduce the thin shell's equation of motion, i.e.,

$$
\dot{a}^{2}+V(a)=0 \text {. }
$$

The potential $V(a)$ is given by

$$
V(a)=F(a)-\left(\frac{m_{s}}{2 a}\right)^{2}-\left(\frac{a G}{m_{s}}\right)^{2},
$$

where $m_{s}(a)=4 \pi a^{2} \sigma$ is the mass of the thin shell. The factors $F(a)$ and $G(a)$, introduced for computational convenience, are defined by

$$
\begin{aligned}
& F(a) \equiv \overline{e^{-2 \beta}}=\frac{1}{2}\left(e^{-2 \beta_{-}}+e^{-2 \beta_{+}}\right), \\
& G(a) \equiv-\frac{1}{2}\left[e^{-2 \beta}\right]_{-}^{+}=\frac{1}{2}\left(e^{-2 \beta_{-}}-e^{-2 \beta_{+}}\right),
\end{aligned}
$$

respectively.

Linearizing around a static solution situated at $a_{0}$, we consider a Taylor expansion of $V(a)$ around $a_{0}$ to second order, given by

$$
V(a)=V\left(a_{0}\right)+V^{\prime}\left(a_{0}\right)\left(a-a_{0}\right)+\frac{1}{2} V^{\prime \prime}\left(a_{0}\right)\left(a-a_{0}\right)^{2}+O\left[\left(a-a_{0}\right)^{3}\right] .
$$

Note that one presumes that $V\left(a_{0}\right)=0$, which is imposed in order to make the expansion consistent. The first and second derivatives of $V(a)$ are, respectively, given by

$$
\begin{gathered}
V^{\prime}(a)=F^{\prime}-2\left(\frac{m_{s}}{2 a}\right)\left(\frac{m_{s}}{2 a}\right)^{\prime}-2\left(\frac{a G}{m_{s}}\right)\left(\frac{a G}{m_{s}}\right)^{\prime}, \\
V^{\prime \prime}(a)=F^{\prime \prime}-2\left[\left(\frac{m_{s}}{2 a}\right)^{\prime}\right]^{2}-2\left(\frac{m_{s}}{2 a}\right)\left(\frac{m_{s}}{2 a}\right)^{\prime \prime}-2\left[\left(\frac{a G}{m_{s}}\right)^{\prime}\right]^{2}-2\left(\frac{a G}{m_{s}}\right)\left(\frac{a G}{m_{s}}\right)^{\prime \prime} .
\end{gathered}
$$

Evaluated at the static solution, at $a=a_{0}$, through a long and tedious calculation, we find $V\left(a_{0}\right)=0$ and $V^{\prime}\left(a_{0}\right)=0$. Thus, the potential, equation (41), reduces to $V(a)=\frac{1}{2} V^{\prime \prime}\left(a_{0}\right)\left(a-a_{0}\right)^{2}+O\left[\left(a-a_{0}\right)^{3}\right]$. From the condition $V^{\prime}\left(a_{0}\right)=0$, one extracts the following useful equilibrium relationship

$$
\left(\frac{m_{s}}{2 a_{0}}\right)^{\prime} \equiv \Gamma=\left(\frac{a_{0}}{m_{s}}\right)\left[F^{\prime}-2\left(\frac{a_{0} G}{m_{s}}\right)\left(\frac{a_{0} G}{m_{s}}\right)^{\prime}\right] .
$$

Note that if $V^{\prime \prime}\left(a_{0}\right)<0$ is verified, the potential $V(a)$ has a local maximum at $a_{0}$, where a small perturbation in the surface radius will produce an irreversible contraction or expansion of the shell. Therefore, the solution is stable if and only if $V(a)$ has a local

minimum at $a_{0}$ and $V^{\prime \prime}\left(a_{0}\right)>0$ is verified. The latter stability condition takes the form

$$
\left(\frac{m_{s}}{2 a}\right)\left(\frac{m_{s}}{2 a}\right)^{\prime \prime}<\Psi-\Gamma^{2}
$$

where $\Psi$ is defined as

$$
\Psi=\frac{F^{\prime \prime}}{2}-\left[\left(\frac{a G}{m_{s}}\right)^{\prime}\right]^{2}-\left(\frac{a G}{m_{s}}\right)\left(\frac{a G}{m_{s}}\right)^{\prime \prime} .
$$




\subsection{The master equation}

Substituting equation (32) into equation (45), one deduces the master equation given by

$$
\sigma^{\prime} m_{s} \eta_{0}>\Theta
$$

where $\eta_{0}=\eta\left(a_{0}\right)$ and $\Theta$ is defined as

$$
\Theta \equiv \frac{a_{0}}{2 \pi}\left(\Gamma^{2}-\Psi\right)+\frac{1}{4 \pi} m_{s} \bar{\Upsilon} .
$$

Now, from the master equation we deduce that the stable equilibrium regions are dictated by the following inequalities

$$
\begin{array}{lll}
\eta_{0}>\bar{\Theta}, & \text { if } & \sigma^{\prime} m_{s}>0, \\
\eta_{0}<\bar{\Theta}, & \text { if } & \sigma^{\prime} m_{s}<0,
\end{array}
$$

with the definition

$$
\bar{\Theta} \equiv \frac{\Theta}{\sigma^{\prime} m_{s}}
$$

In the specific cases that follow, the explicit form of $\bar{\Theta}$ may become extremely messy, so that as in [27, we find it more instructive to show the stability regions graphically.

\section{A dynamic shell around a black hole}

Consider that the interior and exterior spacetimes are given by equation (11), with the following definitions

$$
e^{2 \alpha_{ \pm}}=e^{-2 \beta_{ \pm}}=\left(1-\frac{2 m_{ \pm}}{r_{ \pm}}\right) \text {. }
$$

with $m_{+}=M$ and $m_{-}=m$. These are separated by a thin shell with the surface stresses given by

$$
\begin{aligned}
& \sigma=-\frac{1}{4 \pi a}\left(\sqrt{1-\frac{2 M}{a}+\dot{a}^{2}}-\sqrt{1-\frac{2 m}{a}+\dot{a}^{2}}\right), \\
& \mathcal{P}=\frac{1}{8 \pi a}\left(\frac{1-\frac{M}{a}+\dot{a}^{2}+a \ddot{a}}{\sqrt{1-\frac{2 M}{a}+\dot{a}^{2}}}-\frac{1-\frac{m}{a}+\dot{a}^{2}+a \ddot{a}}{\sqrt{1-\frac{2 m}{a}+\dot{a}^{2}}}\right),
\end{aligned}
$$

with the junction radius at $a>2 M$.

For this case the transparency condition holds, as $T_{\hat{t} \hat{t}}=T_{\hat{r} \hat{r}}=0$ and consequently $\Xi=0$, so that the conservation identity provides the following simple relationship

$$
\sigma^{\prime}=-\frac{2}{a}(\sigma+\mathcal{P})
$$

Taking into account equations (53)-(54), then equation (55) takes the form

$$
\sigma^{\prime}=\frac{1}{4 \pi a^{2}}\left(\frac{1-\frac{3 M}{a}+\dot{a}^{2}-a \ddot{a}}{\sqrt{1-\frac{2 M}{a}+\dot{a}^{2}}}-\frac{1-\frac{3 m}{a}+\dot{a}^{2}-a \ddot{a}}{\sqrt{1-\frac{2 m}{a}+\dot{a}^{2}}}\right),
$$


and at the static solution $a_{0}$ reduces to

$$
\sigma^{\prime}\left(a_{0}\right)=\frac{1}{4 \pi a_{0}^{2}}\left(\frac{1-\frac{3 M}{a_{0}}}{\sqrt{1-\frac{2 M}{a_{0}}}}-\frac{1-\frac{3 m}{a_{0}}}{\sqrt{1-\frac{2 m}{a_{0}}}}\right),
$$

which plays a fundamental role in determining the stability regions.

Evaluated at the static solution, $a_{0}$, equations (53)-(54) reduce to

$$
\begin{aligned}
& \sigma=-\frac{1}{4 \pi a_{0}}\left(\sqrt{1-\frac{2 M}{a_{0}}}-\sqrt{1-\frac{2 m}{a_{0}}}\right), \\
& \mathcal{P}=\frac{1}{8 \pi a_{0}}\left(\frac{1-\frac{M}{a_{0}}}{\sqrt{1-\frac{2 M}{a_{0}}}}-\frac{1-\frac{m}{a_{0}}}{\sqrt{1-\frac{2 m}{a_{0}}}}\right) .
\end{aligned}
$$

The weak energy condition (WEC) holds if $\sigma \geq 0$ and $\sigma+\mathcal{P} \geq 0$ are satisfied, and by continuity implies the null energy condition (NEC), $\sigma+\mathcal{P} \geq 0$. The strong energy condition (SEC) is satisfied if $\sigma+\mathcal{P} \geq 0$ and $\sigma+2 \mathcal{P} \geq 0$. Note that if $m<M$, then the surface energy density is positive, $\sigma>0$, and it can also be shown that the tangential surface pressure is also positive, $\mathcal{P}>0$. Thus, if $m<M$, the NEC, WEC and the SEC are readily satisfied. If $m>M$, then the surface energy density and the surface pressure are negative, consequently violating the energy conditions.

The equation of motion is of the form $\dot{a}^{2}+V(a)=0$. Using the factors of equations (39)-(40), we have

$$
F=1-\frac{m+M}{a} \quad \text { and } \quad G=\frac{M-m}{a},
$$

and the potential is given by

$$
V(a)=1-\frac{M+m}{a}-\frac{m_{s}^{2}}{4 a^{2}}-\frac{(M-m)^{2}}{m_{s}^{2}} .
$$

If $m<M$, then $m_{s}(a)>0$ and $\sigma^{\prime}(a)<0$. If $m>M$, then $m_{s}(a)<0$ and $\sigma^{\prime}(a)>0$. For both cases the master equation $\sigma^{\prime} m_{s} \eta_{0}>\Theta$ is satisfied, so that the stability regions supersede the energy conditions. Thus, equation (48), dictates the following regions of stability

$$
\eta_{0}<\bar{\Theta}
$$

which is shown below the surface plotted in figure 1. This is in agreement with previous results [15, 27].

\section{A dynamic shell around a traversable wormhole}

\subsection{Interior and exterior solutions}

Consider that the interior and exterior spacetimes are given by equation (11), with the following definitions

$$
\begin{aligned}
& e^{2 \alpha_{+}}=e^{-2 \beta_{+}}=\left(1-\frac{2 M}{r_{+}}\right), \\
& \alpha_{-}=\Phi\left(r_{-}\right), \quad e^{-2 \beta_{-}}=\left(1-\frac{b\left(r_{-}\right)}{r_{-}}\right) .
\end{aligned}
$$




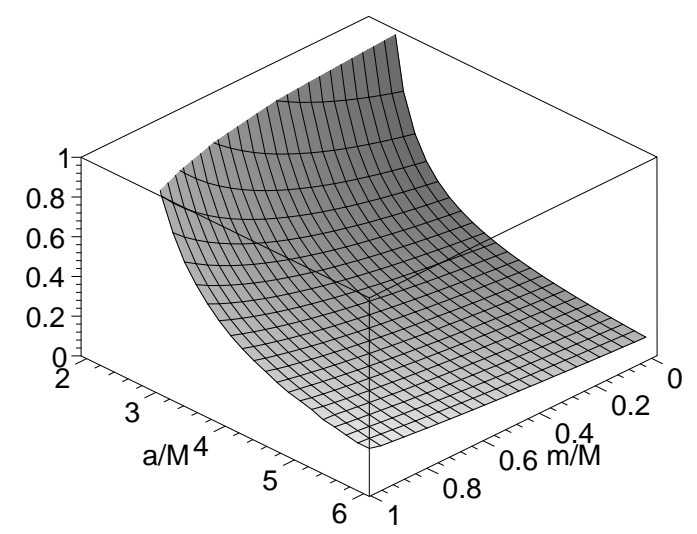

Figure 1. The regions of stability are situated below the surface, which is given by $\bar{\Theta}$. This is in agreement with previous results. See the text for details.

We have considered that the exterior spacetime is given by the Schwarzschild solution. We shall once again drop the $( \pm)$ subscripts, as not to overload the notation. The spacetime with the definition (64) describes a wormhole geometry [19, 32, where $\Phi(r)$ and $b(r)$ are arbitrary functions of the radial coordinate, $r$, denoted as the redshift function and the form function, respectively [32. Note that both solutions are matched at a junction interface, $r=a$, situated outside the event horizon, i.e., $a>r_{b}=2 M$, to avoid a black hole solution.

Relatively to the interior solution, the field equations, eqs. (12)-(14), with the definition (64) provide the following stress-energy scenario

$$
\begin{aligned}
& \rho(r)=\frac{1}{8 \pi} \frac{b^{\prime}}{r^{2}}, \\
& p_{r}(r)=\frac{1}{8 \pi}\left[-\frac{b}{r^{3}}+2\left(1-\frac{b}{r}\right) \frac{\Phi^{\prime}}{r}\right], \\
& p_{t}(r)=\frac{1}{8 \pi}\left(1-\frac{b}{r}\right)\left[\Phi^{\prime \prime}+\left(\Phi^{\prime}\right)^{2}-\frac{b^{\prime} r-b}{2 r(r-b)} \Phi^{\prime}-\frac{b^{\prime} r-b}{2 r^{2}(r-b)}+\frac{\Phi^{\prime}}{r}\right],
\end{aligned}
$$

in which $\rho(r)$ is the energy density; $p_{r}(r)$ is the radial pressure; and $p(r)$ is the transverse pressure.

Wormhole spacetimes necessarily violate the null energy condition (NEC) at the throat. The NEC as defined by equation (15D) now takes the form

$$
T_{\hat{\mu} \hat{\nu}} k^{\hat{\mu}} k^{\hat{\nu}}=\rho(r)+p_{r}(r)=\frac{1}{8 \pi}\left[\frac{b^{\prime} r-b}{r^{3}}+2\left(1-\frac{b}{r}\right) \frac{\Phi^{\prime}}{r}\right] .
$$

Taking into account the flaring out condition of the throat deduced from the mathematics of embedding, i.e., $\left(b-b^{\prime} r\right) / 2 b^{2}>0$, evaluated at the throat $b\left(r_{0}\right)=r=r_{0}$, and due to the finiteness of $\Phi(r)$, we verify that equation (68) is necessarily negative, i.e., $T_{\hat{\mu} \hat{\nu}} k^{\hat{\mu}} k^{\hat{\nu}}<0$. Matter that violates the NEC is denoted as exotic matter.

Recently, Visser, Kar and Dadhich, by implementing the notion of the "volume integral quantifier", showed that the interior wormhole solution may be supported by arbitrarily small quantities of averaged null energy condition (ANEC) violating matter 
33, although the NEC and WEC are always violated for wormhole spacetimes. In the spirit of minimizing the usage of the exotic matter, regions where the surface stress-energy tensor obeys the energy conditions at the junction were found [25, 24]. Recently, it was also shown that traversable wormholes may be supported by phantom energy [30, 31, a null energy condition violating cosmic fluid responsible for the present accelerated expansion of the universe. In Ref. [31, it was shown that these phantom wormholes may be theoretically constructed by vanishing amounts of ANEC violating phantom energy, and the stability regions, in the spirit of this paper, were further explored in Ref. [29].

\subsection{Stability regions}

The surface stresses, eqs. (22) $-(23)$, for this particular case takes the form

$$
\begin{aligned}
& \sigma=-\frac{1}{4 \pi a}\left(\sqrt{1-\frac{2 M}{a}+\dot{a}^{2}}-\sqrt{1-\frac{b(a)}{a}+\dot{a}^{2}}\right), \\
& \mathcal{P}=\frac{1}{8 \pi a}\left(\frac{1-\frac{M}{a}+\dot{a}^{2}+a \ddot{a}}{\sqrt{1-\frac{2 M}{a}+\dot{a}^{2}}}-\frac{\left(1+a \Phi^{\prime}\right)\left(1-\frac{b}{a}+\dot{a}^{2}\right)+a \ddot{a}-\frac{\dot{a}^{2}\left(b-b^{\prime} a\right)}{2(a-b)}}{\sqrt{1-\frac{b(a)}{a}+\dot{a}^{2}}}\right) .
\end{aligned}
$$

The conservation identity provides us with $\sigma^{\prime}=-\frac{2}{a}(\sigma+\mathcal{P})+\Xi$, with $\Xi$ given by

$$
\Xi=-\frac{1}{8 \pi} \frac{\sqrt{1-\frac{b(a)}{a}+\dot{a}^{2}}}{\left(1-\frac{b(a)}{a}\right)}\left[\frac{b^{\prime}(a) a-b(a)}{a^{3}}+2\left(1-\frac{b(a)}{a}\right) \frac{\Phi^{\prime}(a)}{a}\right] .
$$

Equation (30), for this particular case is given by

$$
\sigma^{\prime}=\frac{1}{4 \pi a^{2}}\left(\frac{1-\frac{3 M}{a}+\dot{a}^{2}-a \ddot{a}}{\sqrt{1-\frac{2 M}{a}+\dot{a}^{2}}}-\frac{1-\frac{3 b(a)}{2 a}+\frac{b^{\prime}(a)}{2}+\dot{a}^{2}-a \ddot{a}}{\sqrt{1-\frac{b(a)}{a}+\dot{a}^{2}}}\right),
$$

which at the static solution simplifies to

$$
\sigma^{\prime}\left(a_{0}\right)=\frac{1}{4 \pi a_{0}^{2}}\left(\frac{1-\frac{3 M}{a_{0}}}{\sqrt{1-\frac{2 M}{a_{0}}}}-\frac{1-\frac{3 b\left(a_{0}\right)}{2 a_{0}}+\frac{b^{\prime}\left(a_{0}\right)}{2}}{\sqrt{1-\frac{b\left(a_{0}\right)}{a_{0}}}}\right) .
$$

This relationship will play an important role in determining the stability regions dictated by the master equation, equation (48).

The equation of motion is of the form $\dot{a}^{2}+V(a)=0$, and taking into account the factors of eqs. (39)-(40), which for this case are given by

$$
F=1-\frac{b(a) / 2+M}{a} \quad \text { and } \quad G=\frac{M-b(a) / 2}{a},
$$

the potential takes the form

$$
V(a)=1-\frac{M+\frac{b(a)}{2}}{a}-\frac{m_{s}^{2}}{4 a^{2}}-\frac{\left(M-\frac{b(a)}{2}\right)^{2}}{m_{s}^{2}}
$$

Note that the functions $\Gamma$ and $\Psi$, given by eqs. (44) and (46), respectively, are completely determined by the factors $F, G$ and $m_{s}=4 \pi a^{2} \sigma$. To determine $\bar{\Upsilon}$, given by 
equation (33), and consequently $\Theta$, given by equation (48), one uses the radial derivative of equation (71), and eqs. (69)-(70), evaluated at the static solution. One may now model the wormhole geometry by choosing specific values for the form and redshift functions, and consequently determine the stability regions dictated by the inequalities (49) -(501). As the explicit form of $\bar{\Theta}$ is extremely lengthy, so that as in Ref. [27, 29], we find it more instructive to show the stability regions graphically.

\subsection{Specific form and redshift functions}

We shall in this section consider various choices for the form function, namely $b(r)=r_{0}$ and $b(r)=r_{0}^{2} / r$, and deduce the respective stability regions. We shall verify that the latter may be significantly increased by considering appropriate choices for the redshift function. In this section, we shall relax the condition that the surface energy density be positive, as in considering traversable wormhole geometries, one is already dealing with exotic matter.

5.3.1. $b(r)=r_{0}$. Firstly, consider the particular case of $b(r)=r_{0}$ and $\Phi^{\prime}(a)=\Phi^{\prime \prime}(a)=$ 0 . The redshift function can either be constant, or have the following general form $\Phi(r)=\sum_{n \geq 3} c_{n}(r-a)^{n}$, so that the condition $\Phi^{\prime}(a)=\Phi^{\prime \prime}(a)=0$ is verified. In the analysis that follows below, only particular cases shall be analyzed. Note that the factor $\Phi^{\prime \prime}(a)$ of the model enters into $\Xi^{\prime}$. As we are matching the interior wormhole solution to an exterior vacuum spacetime, then there is no need to impose the condition of an asymptotically flat spacetime. For instance, this notion is reflected in the choice of the redshift function given by $\Phi(r)=(r-a)^{3} / r_{0}^{3}$. However, if one is tempted to model an asymptotically flat wormhole geometry, in the absence of a thin shell, then one may use, for instance, the choice of the redshift function given by $\Phi(r)=r_{0}(r-a)^{3} / r^{4}$. In the analysis that follows we shall separate the cases of $r_{0}<2 M$ and $r_{0}>2 M$, which corresponds to a positive and negative surface energy densities.

For $r_{0}<2 M$, we verify that $m_{s}\left(a_{0}\right)>0$ and $\sigma^{\prime}\left(a_{0}\right)<0$, and consequently $\sigma^{\prime} m_{s}<0$, so that the stability regions are dictated by inequality (50). The stable equilibrium configurations are shown below the surfaces of the plots depicted in figure 2 . In the left plot, we have considered the case of $\Phi(r)=0$. Now, it is interesting to note that one may increase the stability regions by adequately choosing a specific redshift function. To illustrate this, consider for simplicity the choice of $\Phi(r)=r_{0} / r$. Note that qualitatively the stable equilibrium regions, for this case, are increased for high values of $a / M$ as $r_{0} \rightarrow 2 M$, relatively to the $\Phi(r)=0$ case. It is possible to consider more complicated cases, however, this shall not be done here.

For $r_{0}>2 M$, we verify that $m_{s}\left(a_{0}\right)<0$ and $\sigma^{\prime}\left(a_{0}\right)>0$, and the stable equilibrium configurations are also dictated by inequality (50). We have considered the specific cases of $\Phi^{\prime}(a)=\Phi^{\prime \prime}(a)=0$ and $\Phi(r)=-r_{0} / r$, represented in the left and right plots of figure 3, respectively. For both cases the stability regions are also given below the surfaces represented in the plots. Note that the stability regions are significantly increased, for 

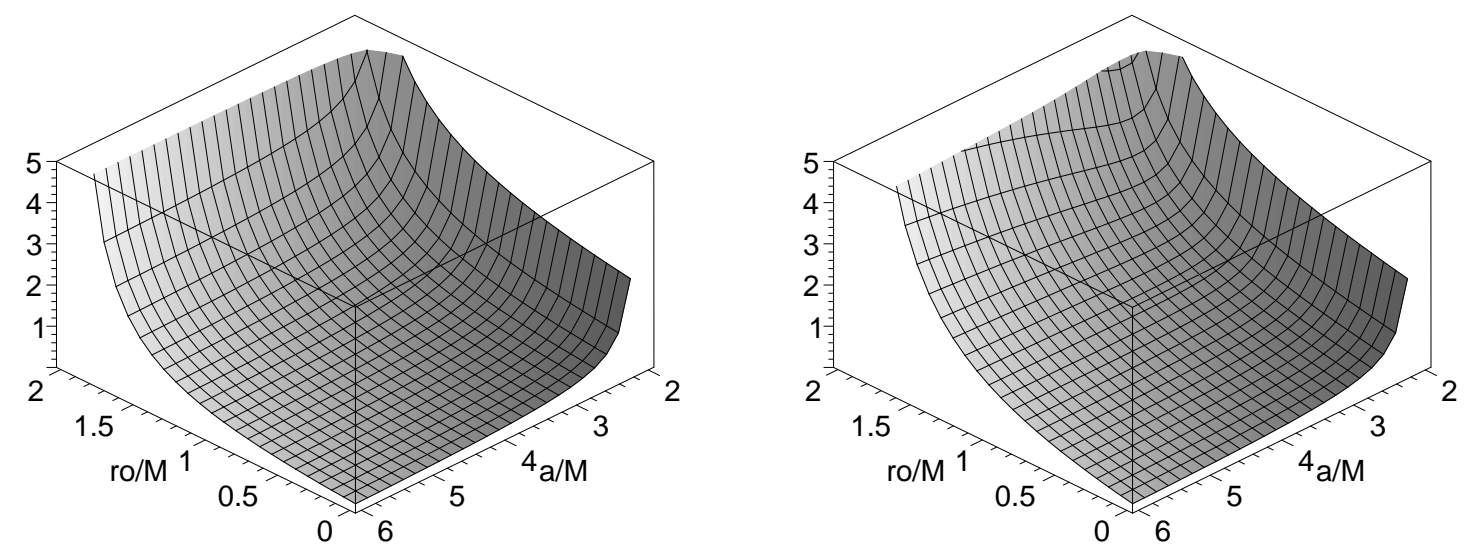

Figure 2. The stable equilibrium regions for the case of $r_{0}<2 M$, with $b(r)=r_{0}$, are represented below the surfaces. We have considered specific choices for the redshift function, namely, $\Phi(r)=0$ and $\Phi(r)=r_{0} / r$, which are represented in the left and right plots, respectively. Note that, qualitatively, the stable equilibrium regions, for $\Phi(r)=r_{0} / r$, are increased relatively to the $\Phi(r)=0$ case. See the text for details.

the $\Phi(r)=-r_{0} / r$ case, as $r_{0} \rightarrow 2 M$, as can be readily verified from figure 3. However, the stability regions for the $\Phi^{\prime}(a)=\Phi^{\prime \prime}(a)=0$ are considerably greater for low values of $M / r_{0}$ and of $a / r_{0}$. Thus, the message that one can extract from this analysis is that one may model stable wormhole geometries, by adequately choosing the redshift function.
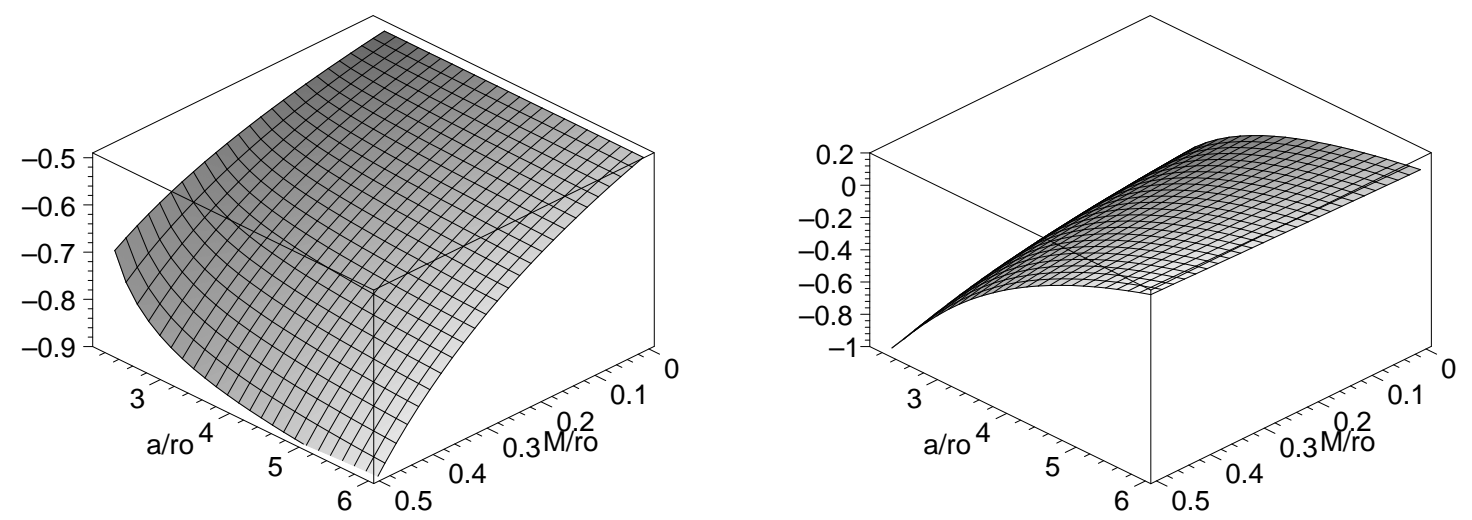

Figure 3. The stable equilibrium regions for the case of $r_{0}>2 M$, with $b(r)=r_{0}$, are represented below the surfaces. We have considered specific choices for the redshift function, namely, $\Phi^{\prime}(a)=\Phi^{\prime \prime}(a)=0$ and $\Phi(r)=-r_{0} / r$, which are represented on the left and right plots, respectively. The stability regions are significantly increased, for the $\Phi(r)=-r_{0} / r$ case, as $r_{0} \rightarrow 2 M$. However, the stability regions for the $\Phi^{\prime}(a)=\Phi^{\prime \prime}(a)=0$ are greater for low values of $M / r_{0}$ and $a / r_{0}$.

5.3.2. $b(r)=r_{0}^{2} / r$. Consider the Ellis drainhole [34, with $b(r)=r_{0}^{2} / r$ and $\Phi(r)=0$. However as in the previous case, this analysis can be extended to an arbitrary redshift function in which $\Phi^{\prime}(a)=\Phi^{\prime \prime}(a)=0$. Recently, in an interesting paper, it was shown that this geometry can also be obtained with 'tachyon matter' as a source term in the field equations and a positive cosmological constant [35]. 
To determine the stability regions of this solution, we shall separate the cases of $b\left(a_{0}\right)<2 M$ and $b\left(a_{0}\right)>2 M$. From equation (69) and the definition of $m_{s}=4 \pi a_{0}^{2} \sigma$, this corresponds to $m_{s}>0$ and $m_{s}<0$, respectively.

For $b\left(a_{0}\right)>2 M$, i.e., for a negative surface energy density, and using the form function considered above, we need to impose the condition $r_{0}>2 M$, so that the junction radius lies outside the event horizon, $a_{0}>2 M$. Thus, the junction radius lies in the following range

$$
r_{0}<a_{0}<\frac{r_{0}^{2}}{2 M}
$$

We verify that $m_{s} \sigma^{\prime}<0$, so that according to the inequality (50), the stability regions lie below the curves depicted in figure 4. We have considered the cases of $r_{0} / M=2.2$ and $r_{0} / M=3$, where the ranges of $a_{0}$ are given by $2.2<a_{0} / M<2.42$ and $3<a_{0} / M<4.5$, respectively. Note that the values of $\eta_{0}$ are negative in the stability regions, however, by increasing the parameter $r_{0} / M$, for $\Phi(r)=0$, the values of $\eta_{0}$ become less restricted, so that the stability regions are increased.

Now, considering a fixed value of $r_{0} / M$ and for $\Phi(r)=0$, considering the choice of $\Phi(r)=r_{0} / r$, we have certain regions for which the stability increases. We have also included the specific choice of $\Phi(r)=r / r_{0}$, for instructive purposes, representing a specific case for which the stable equilibrium configurations decrease.
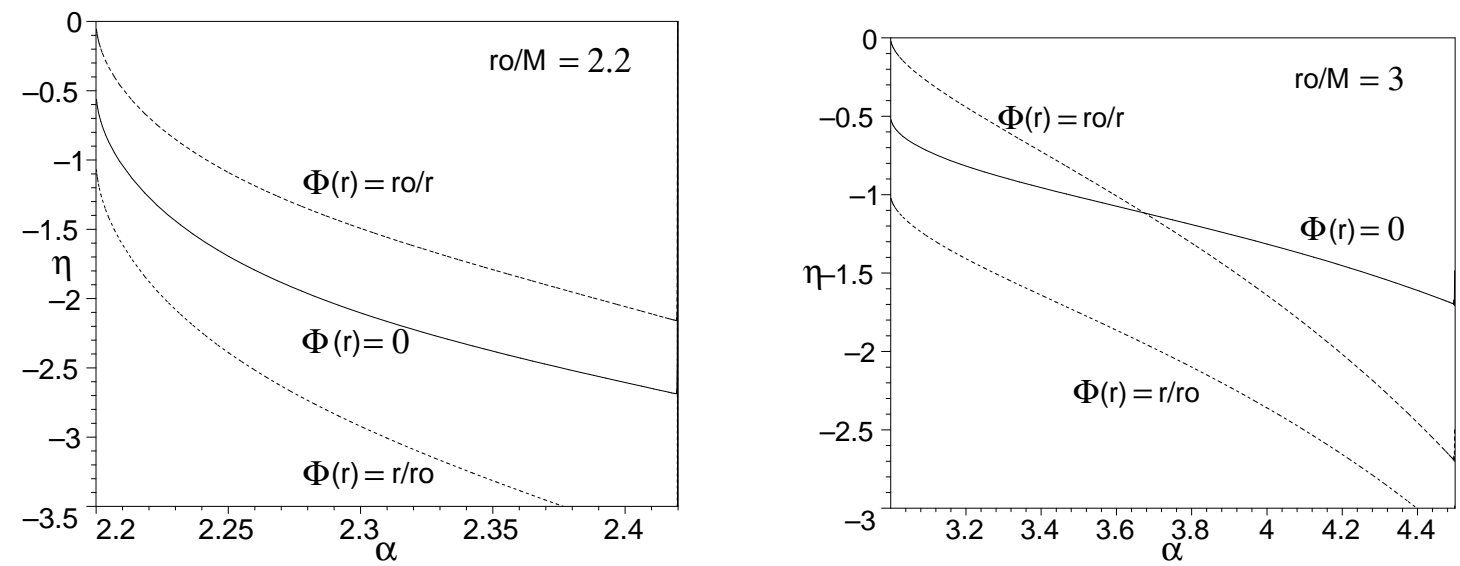

Figure 4. We have defined $\alpha=a_{0} / M$ and considered the specific cases of $r_{0} / M=2.2$ and $r_{0} / M=3$. The stability regions are depicted below the curves. For low values of $r_{0} / M$, we verify that considering the choice of $\Phi(r)=r_{0} / r$, the stability regions increase relatively to $\Phi(r)=0$. For instructive purposes, we have included the case of $\Phi(r)=r / r_{0}$, for which the stability regions decrease. See the text for details.

For $b\left(a_{0}\right)<2 M$, i.e., for a positive surface energy density, and using the form function considered above, we shall separate the cases of $r_{0}<2 M$ and $r_{0}>2 M$.

Firstly, considering the case of $r_{0}>2 M$, we verify that the junction radius lies in the following range

$$
a_{0}>\frac{r_{0}^{2}}{2 M}
$$


For this specific case, $\sigma^{\prime}$ possesses one real positive root, $R$, in the range of equation (177), signalling the presence of an asymptote, $\left.\sigma^{\prime}\right|_{R}=0$. We verify that $\sigma^{\prime}>0$ for $r_{0}^{2} /(2 M)<a_{0}<R$, and $\sigma^{\prime}<0$ for $a_{0}>R$. Thus, the stability regions are given by

$$
\begin{aligned}
& \eta_{0}>\bar{\Theta}, \quad \text { if } \quad \frac{r_{0}^{2}}{2 M}<a_{0}<R, \\
& \eta_{0}<\bar{\Theta}, \quad \text { if } \quad a_{0}>R \text {. }
\end{aligned}
$$

Consider the particular cases of $r_{0} / M=2.2$, so that $a_{0} / M>2.42$, and $r_{0} / M=3$, where $a_{0} / M>4.5$. The asymptotes, $\left.\sigma^{\prime}\right|_{R}=0$, for these cases exist at $R / M \simeq 3.285$ and $R / M \simeq 6.50265$, respectively. These cases are represented in figure 5 We verify that, for $\Phi(r)=0$, and considering a fixed high value of $r_{0} / M$, the specific choice of $\Phi(r)=r_{0} / r$ increases the regions of stability. However, for low values of the parameter $r_{0} / M$, and for low $a_{0} / M$, we need to be more careful, as certain regions for the specific case of $\Phi(r)=r_{0} / r$, decrease the stable equilibrium configurations of the solution, as is qualitatively represented in the right plot of figure 5 .
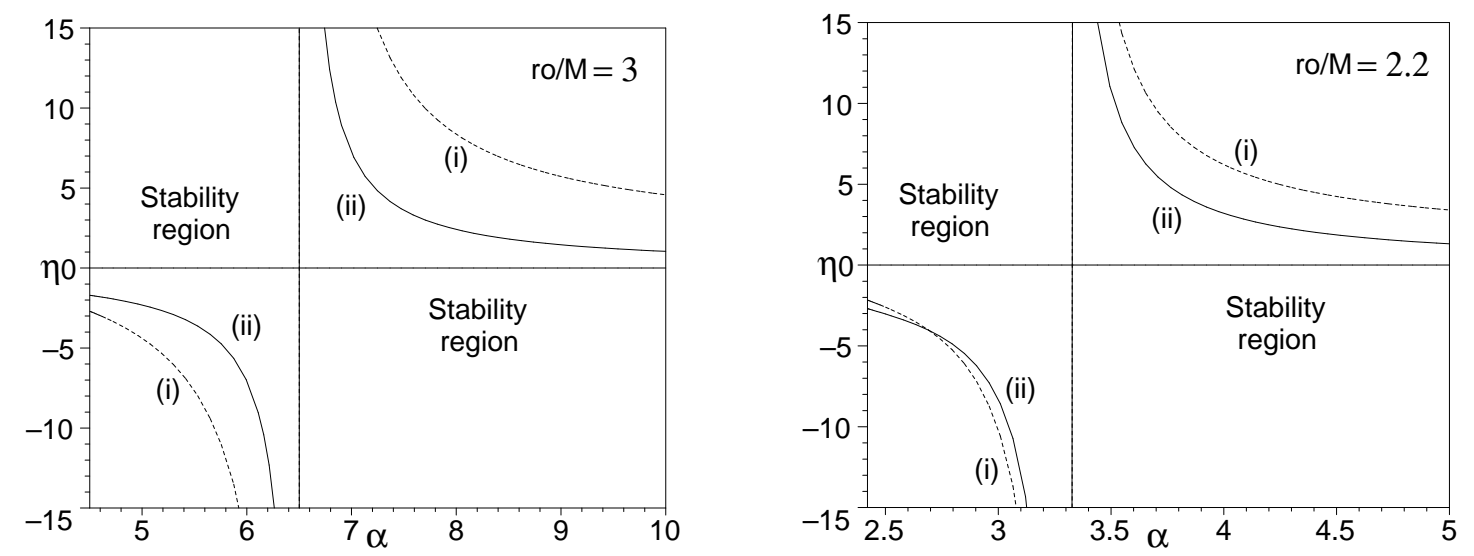

Figure 5. We have defined $\alpha=a_{0} / M$, and considered the cases of $r_{0} / M=3$ and $r_{0} / M=2.2$. The stability regions are depicted in the plots. (i) corresponds to $\Phi(r)=r_{0} / r ;(i i)$ corresponds to $\Phi(r)=0$. Note that one may increase the stability regions by choosing an appropriate redshift function. See the text for details.

Considering the case of $r_{0}<2 M$, we verify that the junction radius lies in the range $a_{0}>2 M$. We have $m_{s} \sigma^{\prime}<0$, so that the stability region lies below the curves depicted in figure 6. Adopting a conservative point of view, we verify that for this case of a positive surface energy density, stability regions exist well within the range $0<\eta_{0} \leq 1$. The stability regions are increased by increasing the value of $r_{0} / M$, with $\Phi(r)=0$, and is further increased by considering the specific choice of $\Phi(r)=r_{0} / r$.

\section{Conclusion}

Thin shells play an extremely fundamental role in general relativity, and have numerous applications ranging from the study of gravitational collapse to the Randall-Sundrum brane world scenario [36]. In this paper, we have analyzed the stability of generic 

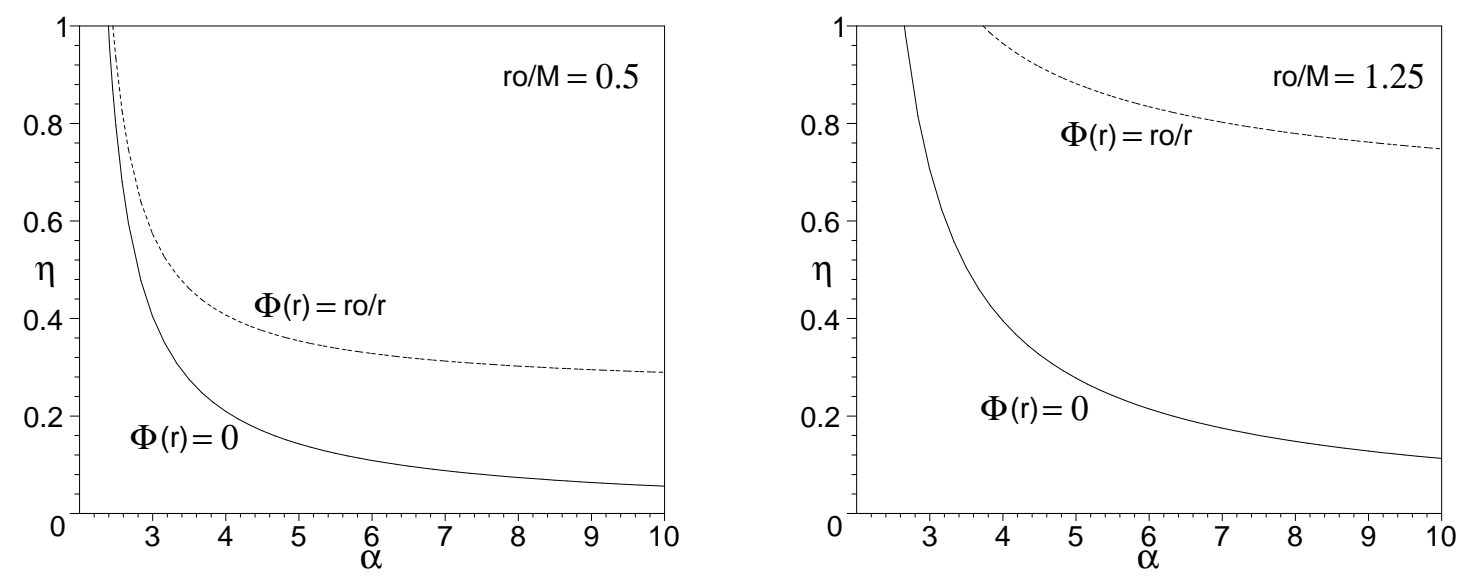

Figure 6. We have defined $\alpha=a_{0} / M$, and considered the specific choices of $r_{0} / M=0.5$ and $r_{=} / M=1.25$. The stability regions are depicted below the curves. Note that the stability regions for $\Phi(r)=0$ are increased by increasing the parameter $r_{0} / M$, and further significantly increased by choosing an appropriate redshift function, for instance, $\Phi(r)=r_{0} / r$. See the text for details.

spherically symmetric thin shells to linearized perturbations around static solutions. We have included the momentum flux term in the conservation identity, deduced from the "ADM" constraint and the Lanczos equations. Following the Ishak-Lake analysis, a master equation dictating the stable equilibrium configurations was deduced. Considering the transparency condition, we studied the stability of thin shells around black holes, showing that our analysis is in agreement with previous results. Applying the analysis to traversable wormhole geometries, considering specific choices for the form function, we deduced stability regions, and found that the latter may be significantly increased by considering appropriate choices for the redshift function.

We have analyzed the stability by defining a parameter $\eta$, so that one does not have to define an equation of state of the stresses involved. Normally, $\sqrt{\eta}$ is interpreted as the speed of sound, so that the requirement that the latter does not exceed the speed of light is naturally $0 \leq \eta<1$. Although we have imposed this condition for the analysis concerning thin shells around black holes, this definition cannot be naively applied to stresses that violate the null energy condition, i.e., "exotic matter". Therefore, we have relaxed the range $0 \leq \eta<1$, when considering the stability analysis for thin shells around traversable wormholes, although stability regions do exist for the referred interval. It is also interesting to note, as emphasized in Ref. [25, that thin shells (or domain walls) arise in models with spontaneously broken discrete symmetries in field theory. Note that these models involve a set of real scalar fields $\phi_{i}$, with a Lagrangian of the form $\mathcal{L}=\frac{1}{2}\left(\partial_{\mu} \phi_{i}\right)^{2}-V(\phi)$, where the potential $V(\phi)$ has a discrete set of degenerate minima. Thus, by suitably choosing $\phi_{i}$ and $V(\phi)$, one may obtain the dynamically stable thin shells analyzed in this work. 


\section{References}

[1] N. Sen, "Über die grenzbedingungen des schwerefeldes an unsteig keitsflächen," Ann. Phys. (Leipzig) 73, 365 (1924).

[2] K. Lanczos, "Flächenhafte verteiliung der materie in der Einsteinschen gravitationstheorie," Ann. Phys. (Leipzig) 74, 518 (1924).

[3] G. Darmois, "Mémorial des sciences mathématiques XXV," Fascicule XXV ch V (Gauthier-Villars, Paris, France, 1927).

[4] S. O'Brien and J. L. Synge, Commun. Dublin Inst. Adv. Stud. A., no. 9 (1952).

[5] A. Lichnerowicz, "Théories Relativistes de la Gravitation et de l'Electromagnetisme," Masson, Paris (1955).

[6] W. Israel, "Singular hypersurfaces and thin shells in general relativity," Nuovo Cimento 44B, 1 (1966); and corrections in ibid. 48B, 463 (1966).

[7] J. R. Oppenheimer and H. Snyder, "On Continued Gravitational Contraction," Phys. Rev. 56, 455 (1939).

[8] D. Garfinkle, "Corrections to the thin-wall approximation in general relativity," Phys. Rev. D 41, 1889 (1990).

[9] S. Khakshournia and R. Mansouri, "Dynamics of general relativistic spherically symmetric dust thick shells," Gen. Rel. Grav. 34, 1847 (2002) arXiv:gr-qc/0308025.

[10] C. Barrabès and W. Israel, "Thin shells in general relativity and cosmology: The lightlike limit," Phys. Rev. D 43, 1129 (1991).

[11] R. Mansouri and M. Khorrami, "The equivalence of Darmois-Israel and distributional-metod for thin shells in general relativity," J. Math. Phys. 37, 5672 (1996) arXiv:gr-qc/9608029.

[12] P. Musgrave and K. Lake, "Junctions and thin shells in general relativity using computer algebra I: The Darmois-Israel Formalism," Class. Quant. Grav. 131885 (1996) arXiv:gr-qc/9510052.

[13] V. P. Frolov, M. A. Markov and V. F. Mukhanov, "Black holes as possible sources of closed and semiclosed worlds," Phys. Rev. D 41, 383 (1990).

[14] J. Fraundiener, C. Hoenselaers and W. Konrad, "A shell around a black hole," Class. Quant. Grav. 7, 585 (1990).

[15] P. R. Brady, J. Louko and E. Poisson, "Stability of a shell around a black hole," Phys. Rev. D 44, 1891 (1991).

[16] M. Visser, "Traversable wormholes: Some simple examples," Phys. Rev. D 393182 (1989).

[17] M. Visser, "Traversable wormholes from surgically modified Schwarzschild spacetimes," Nucl. Phys. B 328203 (1989).

[18] M. Visser, "Quantum mechanical stabilization of Minkowski signature," Phys. Lett. B 242, 24 (1990).

[19] M. Visser, Lorentzian Wormholes: From Einstein to Hawking (American Institute of Physics, New York, 1995).

[20] E. Poisson and M. Visser, "Thin-shell wormholes: Linearization stability," Phys. Rev. D 527318 (1995) arXiv:gr-qc/9506083.

[21] E. F. Eiroa and G. E. Romero "Linearized stability of charged thin-shell wormoles," Gen. Rel. Grav. 36 651-659 (2004) arXiv:gr-qc/0303093.

[22] F. S. N. Lobo and P. Crawford, "Linearized stability analysis of thin-shell wormholes with a cosmological constant," Class. Quant. Grav. 21, 391 (2004) arXiv:gr-qc/0311002.

[23] J. P. S. Lemos, F. S. N. Lobo and S. Q. de Oliveira, "Morris-Thorne wormholes with a cosmological constant," Phys. Rev. D 68, 064004 (2003) arXiv:gr-qc/0302049.

[24] F. S. N. Lobo, "Energy conditions, traversable wormholes and dust shells," (to appear in Gen. Rel. Grav.) arXiv:gr-qc/0410087.

[25] F. S. N. Lobo, "Surface stresses on a thin shell surrounding a traversable wormhole," Class. Quant. Grav. 214811 (2004) arXiv:gr-qc/0409018.

[26] J. P. S. Lemos and F. S. N. Lobo, "Plane symmetric traversable wormholes in an anti-de Sitter 
background," Phys. Rev. D 69, 104007 (2004) arXiv:gr-qc/0402099.

[27] M. Ishak and K. Lake, "Stability of transparent spherically symmetric thin shells and wormholes," Phys. Rev. D 65044011 (2002) arXiv:gr-qc/0108058.

[28] S. M. C. V. Gonçalves, "Relativistic shells: Dynamics, horizons, and shell crossing," Phys. Rev. D 66, 084021 (2002) arXiv:gr-qc/0212124.

[29] F. S. N. Lobo, "Stability of phantom wormholes," Phys. Rev. D 71, 124022 (2005) arXiv:gr-qc/0506001.

[30] S. Sushkov, "Wormholes supported by a phantom energy," Phys. Rev. D 71, 043520 (2005) arXiv:gr-qc/0502084.

[31] F. S. N. Lobo, "Phantom energy traversable wormholes," Phys. Rev. D 71, 084011 (2005) arXiv:gr-qc/0502099.

[32] M. Morris and K.S. Thorne, "Wormholes in spacetime and their use for interstellar travel: A tool for teaching General Relativity," Am. J. Phys. 56, 395 (1988).

[33] M. Visser, S. Kar and N. Dadhich, "Traversable wormholes with arbitrarily small energy condition violations," Phys. Rev. Lett. 90, 201102 (2003) arXiv:gr-qc/0301003.

[34] H. G. Ellis, "Ether flow through a drainhole: A particle model in general relativity," J. Math. Phys. 14, 104 (1973).

[35] A. Das and S. Kar, "The Ellis wormhole with 'tachyon matter'," Class. Quant. Grav. 223045 (2005) arXiv:gr-qc/0505124.

[36] The literature is extremely extensive, but for some examples see:

K. Lake and C. Hellaby, "Collapse of gravitational fluid spheres," Phys. Rev. D 24, 3019 (1981);

K. Lake, "Collapse of radiating imperfect fluid spheres," Phys. Rev. D 26, 518 (1982);

A. Vilenkin, "Gravitational field of vacuum domain walls and strings," Phys. Rev. D 23, 852 (1981);

J. Ipser and P. Sikivie, "Gravitationally repulsive domain wall," Phys. Rev. D 30, 712 (1984);

V. A. Berezin, V. A. Kuzmin and I. I. Tkachev, "Dynamics of bubbles in general relativity," Phys. Rev. D 36, 2919 (1987);

R. Balbinot, C. Barrabès and A. Fabbri, "Friedmann universes connected by Reissner-Nordström wormholes," Phys. Rev. D 49, 2801 (19934);

N. Sakai and K. Maeda, "Junction conditions of Friedmann-Robertson-Walker space-times," Phys. Rev. D 50, 5425 (1994) arXiv:gr-qc/9311024;

F. Fayos, J. M. M. Senovilla and R. Torres, "General matching of two spherically symmetric spacetimes," Phys. Rev. D 54, 4862 (1996);

L. Randall L and R. Sundrum, "A large mass hierarchy from a small extra dimension." Phys. Rev. Lett. 83, 3370 (1999) arXiv:hep-ph/9905221;

L. Randall and R. Sundrum, "An alternative to compactification," Phys. Rev. Lett. 83, 4690 (1999) arXiv:hep-th/9906064;

P. Kraus, "Dynamics of anti-de Sitter domain walls," JHEP9912, 011 (1999) arXiv:hep-th/9910149;

N. Kaloper, "Bent domain walls as braneworlds," Phys. Rev. D 60123506 (1999) arXiv:hep-th/9905210;

P. Binétruy, C. Deffayet, U. Ellwanger and D. Langlois, "Brane cosmological evolution in a bulk with cosmological constant," Phys. Lett. B 477285 (2000) arXiv:hep-th/9910219;

L. Anchordoqui and K. Olsen, "Comments on brane world cosmology," Mod. Phys. Lett. A 16 1157 (2001) arXiv:hep-th/0008102;

D. Langlois, K. Maeda and D. Wands, "Conservation Laws for Collisions of Branes and Shells in General Relativity," Phys. Rev. Lett. 88, 181301 (2002);

A. Chamblin, S. W. Hawking and H. S. Reall, "Brane-world black holes," Phys. Rev. D 61 065007 (2000) arXiv:hep-th/9909205;

R. Emparan, R. Gregory, and C. Santos, "Black holes on thick branes," Phys. Rev. D 63, 104022 (2001). 